\title{
High Energy Astrophysical Neutrino Flux Characteristics for Neutrino-induced Cascades Using IC79 and IC86-String IceCube Configurations
}

\section{The IceCube Collaboration ${ }^{\dagger}$,}

$\dagger$ http://icecube.wisc.edu/collaboration/authors/icrc15_icecube

E-mail: hans.niederhausen@stonybrook. edu

\begin{abstract}
We have performed a new measurement of the all-sky diffuse flux of high energy, $E>10 \mathrm{TeV}$, extraterrestrial neutrinos by studying neutrino-induced particle showers (cascades) with IceCube data collected during 641 days in 2010-2012. Cascades arise predominantly in electron and tau neutrino interactions and have good energy resolution, and thus are well-suited for the spectral characterization of the extraterrestrial flux. We have improved upon previous analyses by including high-energy cascades with vertices outside, but close to the detector, thereby enlarging the event sample by up to a factor of two for $E>100 \mathrm{TeV}$. A total of 172 cascades with energies ranging from $10 \mathrm{TeV}$ to $1 \mathrm{PeV}$ have been observed, of which approximately $60 \%$ ( $75 \%$ above $100 \mathrm{TeV}$ ) have not previously been reported by IceCube. The dominant extra-terrestrial component is well described by a smooth and featureless power-law. The preliminary fit result is a soft spectral index of $\gamma=2.67_{-0.13}^{+0.12}$ and a per-flavor normalization at $100 \mathrm{TeV}$ of $\phi=\left(2.3_{-0.6}^{+0.7}\right) \cdot 10^{-18} \mathrm{GeV}^{-1} \mathrm{~s}^{-1} \mathrm{sr}^{-1} \mathrm{~cm}^{-2}$ in agreement with previous IceCube results. The background-only hypothesis is rejected with a significance of $4.7 \sigma$. Finally, we have divided the cascade data according to the neutrino arrival direction into two samples corresponding to the Northern and Southern celestial hemispheres. No spectral difference was found $\left(\gamma_{\text {north }}=2.69_{-0.34}^{+0.34}, \phi_{\text {north }}=1.7_{-1.2}^{+1.3} \mathrm{GeV}^{-1} \mathrm{~s}^{-1} \mathrm{sr}^{-1} \mathrm{~cm}^{-2}, \gamma_{\text {south }}=2.68_{-0.22}^{+0.20}\right.$, $\phi_{\text {south }}=1.9_{-0.6}^{+0.8} \mathrm{GeV}^{-1} \mathrm{~s}^{-1} \mathrm{sr}^{-1} \mathrm{~cm}^{-2}$ ), so that the extraterrestrial neutrino fluxes originating from the Northern and Southern hemispheres are consistent.
\end{abstract}

Corresponding authors: Hans Niederhausen ${ }^{1 *}$, Mariola Lesiak-Bzdak ${ }^{1}$, Achim Stoess $^{2}$

${ }^{1}$ Department of Physics and Astronomy, Stony Brook University, Stony Brook, NY 11794-3800

${ }^{2}$ DESY-Zeuthen, D-15735 Zeuthen, Germany

The 34th International Cosmic Ray Conference,

30 July- 6 August, 2015

The Hague, The Netherlands

\footnotetext{
*Speaker.
} 
Introduction

IceCube is a cubic-kilometer neutrino detector installed in the glacial ice at the geographic South Pole [1] between depths of $1450 \mathrm{~m}$ and $2450 \mathrm{~m}$. IceCube observes neutrinos based on optical measurements of Cherenkov radiation emitted by secondary particles produced in neutrino interactions in the surrounding ice or the nearby bedrock. Those interaction are dominated by deep-inelastic scattering (DIS) of neutrinos off nucleons in the ice. Events are classified according to the topology of their light deposition with tracks and cascades being the main signatures. Tracks arise primarily from through-going muons while cascades may be produced by charged-current interactions of $v_{e}$ and $v_{\tau}$ and by neutral current interactions of any flavor. They are characterized by their point-like light emission. Various hybrid signatures exist, e.g. $v_{\mu} \mathrm{CC}$ events that start inside the detector appear as cascade with an outgoing track. At highest energies $\left(E_{v}>1 \mathrm{PeV}\right)$ multiple $v_{\tau}$-topologies are possible with the "double-bang" being the most prominent [2]. At trigger level the vast majority of events in IceCube are muons that have been produced in cosmic ray induced air showers. Their rate in IceCube exceeds that of neutrinos by orders of magnitude $\left(\sim 10^{6}\right)$. Atmospheric neutrinos are produced in the same air showers and form the second largest contribution. At energies relevant to this work, conventional atmospheric neutrinos are dominated by $v_{\mu}\left(v_{\mu}: v_{e} \sim 30\right.$ at $\left.E_{v} \sim 10 \mathrm{TeV}\right)$ that stem primarily from the decay of charged kaons $\left(K^{ \pm}\right)$and charged pions $\left(\pi^{ \pm}\right)$. Conventional $v_{e}$ instead come equally from the decay of charged and neutral kaons $\left(K^{ \pm, 0}\right)$. Those light mesons experience energy loss before they decay and thus the conventional neutrino spectrum follows $\sim E^{-3.7}$. 'Prompt' atmospheric neutrinos are associated with the decay of heavier mesons involving charm quarks and thus produce an equal admixture of $v_{\mu}$ and $v_{e}$. Due to the very small lifetime of those mesons the corresponding prompt spectrum mimics that of primary cosmic rays, i.e. $\sim E^{-2.7}$. The cascade channel benefits from the small conventional atmospheric background levels compared to the track channel. In addition fully contained cascades with vertices well within the instrumented volume have superior (deposited) energy resolution of $\sim 15 \%$ at relevant energies since for such events the detector acts as a calorimeter. Partially contained cascades that start beyond the detector boundary can be reconstructed with an average energy resolution of $\sim 30 \%$.

IceCube recently reported the discovery of an all-sky diffuse flux of high energy neutrinos $\left(E_{v}>\right.$ $60 \mathrm{TeV}$ ) [3] from yet unresolved sources with an intensity almost at the level of the WaxmanBahcall bound [4]. It was found to be well described by a single power-law with spectral index $\gamma=2.3 \pm 0.3$ [3]. Subsequently an extension of this analysis to lower energies concluded $\gamma=2.46 \pm 0.12\left(E_{V}>1 \mathrm{TeV}\right)$ [5]. Astrophysical neutrino production is generally assumed to be associated with the acceleration of cosmic rays at their sources. Detailed predictions of the neutrino flux characteristics are source specific [6] and at present those neutrino sources still escape experimental detection. Based on diffusive shock acceleration and neutrino oscillations over astrophysical relevant distances one expects the astrophysical neutrino flux to exhibit a power-law spectrum [7] with an approximately equal flavor admixture at earth $\left(v_{e}: v_{\mu}: v_{\tau} \approx 1: 1: 1\right)$ [8]. This expectation, especially the flavor ratio, is modified when more complex source environments are considered [9]. Furthermore the flux is expected to be isotropic if many individually weak sources contribute to the flux. The current IceCube measurements focusing on "starting events" (tracks and cascades) of all neutrino flavors and interactions are consistent with these expectations $[3,5,10]$. The astrophysical flux has also been observed using $v_{\mu}$-tracks from the northern celestial hemisphere. A spectral index of $2.2 \pm 0.2$ [11] was found to be in agreement with the earlier 

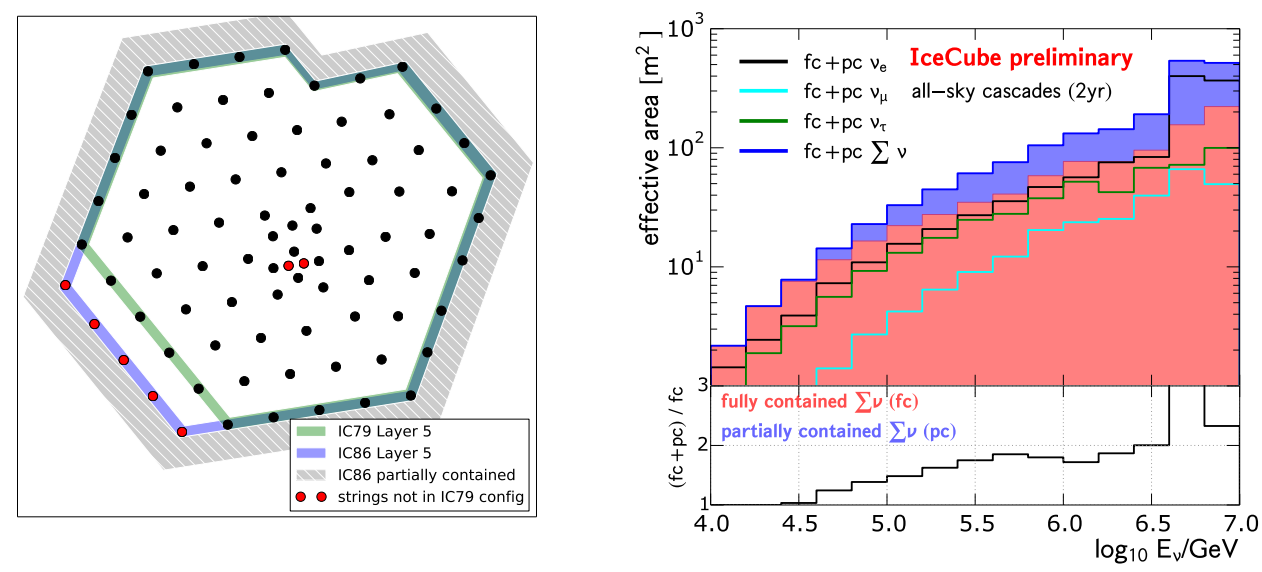

Figure 1: Cross-sectional visualization of the detector in the $x, y$-plane (left); 2-year effective area of this event selection for combined fully and partially contained cascades (right).

"starting event" results $[3,5,10]$.

In this paper we present the first results on the astrophysical neutrino induced cascades flux characteristics using 641 days of IceCube data taken from May 2010 to May 2012. The analysis is based on the event topology selection criteria of ref. [12] to select high energy cascades $(\geq 10 \mathrm{TeV})$. We achieved an improved sensitivity to cascades compared to earlier analyses by including cascades which are partially contained in the detector volume (in addition to cascades which are fully contained), thus significantly enhancing this cascade sample in the background free region above $100 \mathrm{TeV}$ (see Fig. 1). The final event selection, optimized for maximum signal significance, retained 172 (contained: 152, partially contained: 20) cascades with energies greater than $10 \mathrm{TeV}$. From Monte Carlo simulations we estimated a neutrino purity of $90 \%$ (atmospheric and astrophysical $v$ ). The neutrino effective area is shown in Fig. 1 (right). Fig. 2 shows the distribution of the "DelayTime" variable used in the event selection at a level where the data is dominated by atmospheric muon background. It measures the time difference between the time of the first observed photon in the event and the earliest possible time for that photon to stem from the reconstructed cascade (causality). The data is well described by the Monte Carlo simulations. The CORSIKA [13] software package was used to generate cosmic ray background according to the composition model of ref. [14]. For the simulation of neutrino propagation and interaction the neutrino-generator package is used. The atmospheric neutrino flux has been calculated according to the HKKMS06 [15] (conventional $v$ ) and ERS [16] (prompt $v$ ) models, modified and extrapolated according to the expected dampening of the neutrino flux due to the presence of the knee in the cosmic ray energy spectrum [17]. For contained cascades originating in the IceCube instrumented volume (see Fig. 1 (left)) we further take into account the "self-veto" effect of refs. [18]. The baseline model for astrophysical neutrinos is a single power-law with normalization parameter $\phi$ at $E_{v}=100 \mathrm{TeV}$ and $\gamma$ being the spectral index parameter: $\Phi_{v}=\phi \times\left(E_{v} / 100 \mathrm{TeV}\right)^{-\gamma}$. The measurement of the astrophysical neutrino spectrum is performed by matching the reconstructed 

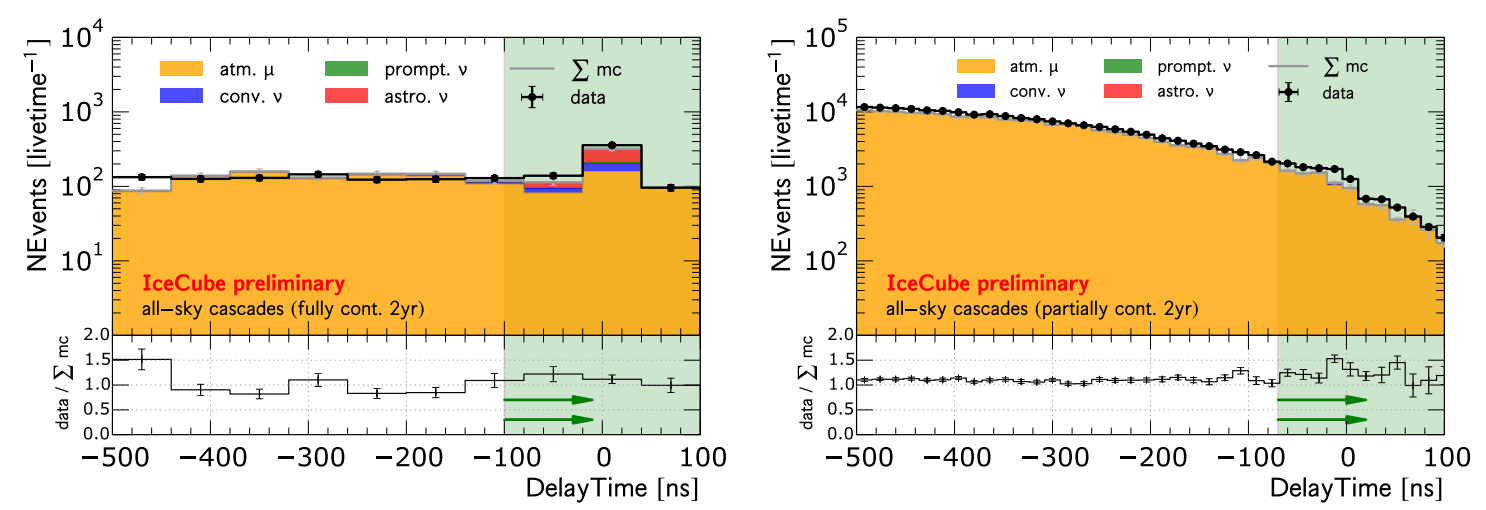

Figure 2: Distribution of the "DelayTime" for a $\mu$-background dominated event selection. Left: contained sample; Right: partially contained sample. Signal region shown in green. Neutrino expectations according to fit result to the final event sample.

deposited energy distribution to the simulation prediction using frequentist methods based on maximum likelihood.

Analysis Method

In order to make an inference about the astrophysical flux from the astrophysical component in this cascade sample we employ the method of maximum likelihood, similar to previous IceCube analyses $[17,3,5,10]$. We separate the contained cascade events according to their reconstructed zenith angle $\Theta_{\text {reco }}$ into two groups: 'Northern sky $\left(\cos \Theta_{\text {reco }}<0\right)$ and 'Southern sky' $\left(\cos \Theta_{\text {reco }}>=0\right)$ ). We then consider the all-sky partially contained cascade sample as the third group. For each group we obtain a frequency distribution of the reconstructed deposited energy by binning its values into $N$ bins. This allows us to construct the following standard poisson profile likelihood function for a joint fit of the three groups

$$
L\left(\boldsymbol{\theta}_{\boldsymbol{r}} \mid \underline{n}\right)=\underset{\boldsymbol{\theta}_{\boldsymbol{s}}}{\operatorname{argmax}} L\left(\boldsymbol{\theta}_{\boldsymbol{r}}, \boldsymbol{\theta}_{\boldsymbol{s}} \mid \underline{n}\right)=\underset{\boldsymbol{\theta}_{\boldsymbol{s}}}{\operatorname{argmax}} \prod_{i=1}^{3} \prod_{j=1}^{N} \frac{\mu_{i j}\left(\boldsymbol{\theta}_{\boldsymbol{r}}, \boldsymbol{\theta}_{\boldsymbol{s}}\right)^{n_{i j}}}{n_{i j} !} e^{-\mu_{i j}\left(\boldsymbol{\theta}_{\boldsymbol{r}}, \boldsymbol{\theta}_{\boldsymbol{s}}\right)}
$$

where $n_{i j}$ is the observed number of events in the $i j$-th bin, while $\boldsymbol{\theta}_{\boldsymbol{r}}, \boldsymbol{\theta}_{\boldsymbol{s}}$ are vectors of physics parameters and nuisance parameters to include systematic uncertainties, respectively. The expected number of events in the $i j$-th bin, $\mu_{i j}\left(\boldsymbol{\theta}_{\boldsymbol{r}}, \boldsymbol{\theta}_{\boldsymbol{s}}\right)=\mu_{i j}^{a t m . \mu}+\mu_{i j}^{a t m . v}+\mu_{i j}^{a s t r o . v}$, is a superposition of the different models described in the previous section. The maximum likelihood estimate $\hat{\boldsymbol{\theta}}_{\boldsymbol{r}}$ is obtained numerically by minimizing $-2 \log L\left(\boldsymbol{\theta}_{\boldsymbol{r}} \mid \underline{n}\right)$ with respect to $\boldsymbol{\theta}_{\boldsymbol{r}}$. We construct approximate confidence intervals and regions with asymptotic coverage using Wilk's theorem [19]. Here we consider $\phi$ and $\gamma$ as physics parameters and the flux normalizations of the neutrino backgrounds $\phi_{\text {conv }}, \phi_{\text {prompt }}$ as well as an energy related scaling parameter $\varepsilon$ as nuisance parameters. Those nuisance parameters $\boldsymbol{\theta}_{s}$ contribute additional additive penalty terms $\log \left(L_{s y s}\right)=\sum_{s}\left(\theta_{s}-\theta_{s, 0}\right)^{2} / \sigma_{\theta_{s}}^{2}$ to the likelihood function that reflect the associated systematic uncertainties $\sigma_{\theta_{s}}$. The fit is performed in the energy range from $\log _{10} E / \mathrm{GeV}=4.1$ to $\log _{10} E / \mathrm{GeV}=6.9$. There are two main sources of detector related systematic uncertainties. The in-situ sensitivity of the IceCube Digital Optical Module (DOM), dominated by the absolute efficiency of its PMT, has a relative uncertainty of 10\% [21]. Additionally, in order to model the 


\begin{tabular}{lcc}
\hline Detector Syst. & Value & $\hat{\varepsilon}$ \\
\hline DOM & $-10 \%$ & 1.11 \\
DOM & $+10 \%$ & 0.91 \\
Scatt. & $+10 \%$ & 1.11 \\
Abs. & $+10 \%$ & 1.11 \\
Scatt./Abs. & $-7 \%$ & 0.96 \\
\hline Combined $\varepsilon_{\text {tot }}$ & \multicolumn{2}{l}{$1.00 \pm 0.15$} \\
\hline
\end{tabular}

\begin{tabular}{lll}
\hline Par & Prior & Result \\
\hline$\gamma$ & - & $2.67_{-0.13}^{+0.12}$ \\
$\phi$ & - & $2.3_{-0.6}^{+0.7} \mathrm{c.u}$. \\
$\phi_{\text {conv }}$ & $1.0 \pm 0.3$ & $0.85_{-0.29}^{+0.28} \cdot \Phi_{H K K M S 06}$ \\
$\phi_{\text {prompt }}$ & $1_{-1}^{+3}$ & $0.5_{-0.5}^{+2.2} \cdot \Phi_{\text {ERS }}$ \\
$\varepsilon$ & $1.00 \pm 0.15$ & $0.96_{-0.15}^{+0.14}$ \\
\hline with 1 c.u. & $\equiv 10^{-18} \mathrm{GeV}^{-1} \mathrm{~s}^{-1}$ & $\mathrm{sr}^{-1} \mathrm{~cm}^{-2}$ \\
\hline
\end{tabular}

Table 1: Summary of systematics evaluation (left); summary of fit results (right).

photon propagation in the glacial ice at the South Pole we rely on a measurement of scattering and absorption using IceCube's LED calibration system. The associated uncertainty is $10 \%$ [22]. The influence of those effects on this analysis has been studied using dedicated simulations with different DOM efficiencies $(-10 \%,+10 \%)$ and variations of the scattering and absorption of the ice $\left(\Delta \alpha_{\text {scatt }}:+10 \%, \Delta \alpha_{a b s}:+10 \%\right.$, both : $\left.-7 \%\right)$. In order to account for those uncertainties in the likelihood method, a nuisance parameter $\varepsilon$ that effectively translates the model predictions to lower $(\varepsilon<1)$ or higher $(\varepsilon>1)$ reconstructed energies was added. The result of a dedicated simulation study is summarized in Table 1 (left). We find that the DOM efficiency uncertainty and the ice model uncertainty each translate into a $10 \%$ uncertainty on $\varepsilon$. By adding them in quadrature we conclude that detector related systematics are well accounted for by including $\varepsilon=1.00 \pm 0.15$ into the likelihood function. The average uncertainty of the expected conventional atmospheric neutrino flux at energies relevant for this work is 30\% [20]. Uncertainties of the prompt atmospheric neutrino flux are model dependent and no experimental evidence for this flux exists. An upper limit of $\phi_{\text {prompt }}<2.4(<3.8)$ at $68 \%$ C.L. (90\% C.L.) has been obtained in ref. [17] based on the ERS model. Accordingly, we assume a systematic uncertainty of $300 \%$ on the normalization of the prompt ERS model.

Results The observed reconstructed energy distributions of all events that pass the cascade selection criteria of this work are shown in Fig. 3 and compared to the expectation according to the best-fit single, unbroken power-law: $\phi=\left(2.3_{-0.6}^{+0.7}\right) \cdot 10^{-18} \mathrm{GeV}^{-1} \mathrm{~s}^{-1} \mathrm{sr}^{-1} \mathrm{~cm}^{-2}$ and $\gamma=2.67_{-0.13}^{+0.12}$. A good description of the data is achieved as evidenced by the corresponding goodness-of-fit (g.o.f) p-value of 0.32. This is based on the g.o.f test-statistic described in ref. [23] for which we obtained the sampling distribution from parametric bootstrap ("toy experiments") assuming the best-fit. The approximate $68 \%$ confidence region (red) for the astrophysical parameters is shown in Fig. 4 (right). We find this cascade sample to be dominated by astrophysical neutrinos $(\sim 65 \%)$ over the entire energy range. The best-fit values for the remaining parameters are shown in Table 1 (right). When the "background-only" hypothesis $(\phi=0)$ is assumed, an unrealistically large prompt atmospheric neutrino component characterized by $\phi_{\text {prompt }}=7 \cdot \Phi_{E R S}$ is required to describe the data. This however is strongly disfavored w.r.t the best-fit by $4.7 \sigma$ based on the sampling distribution of the likelihood ratio test-statistic $-2 \cdot \log \left(L_{b} / L_{s+b}\right)$ that has obtained assuming the background-only result. The rejection is based on the relative suppression of the prompt flux from the Southern hemisphere compared to the flux 

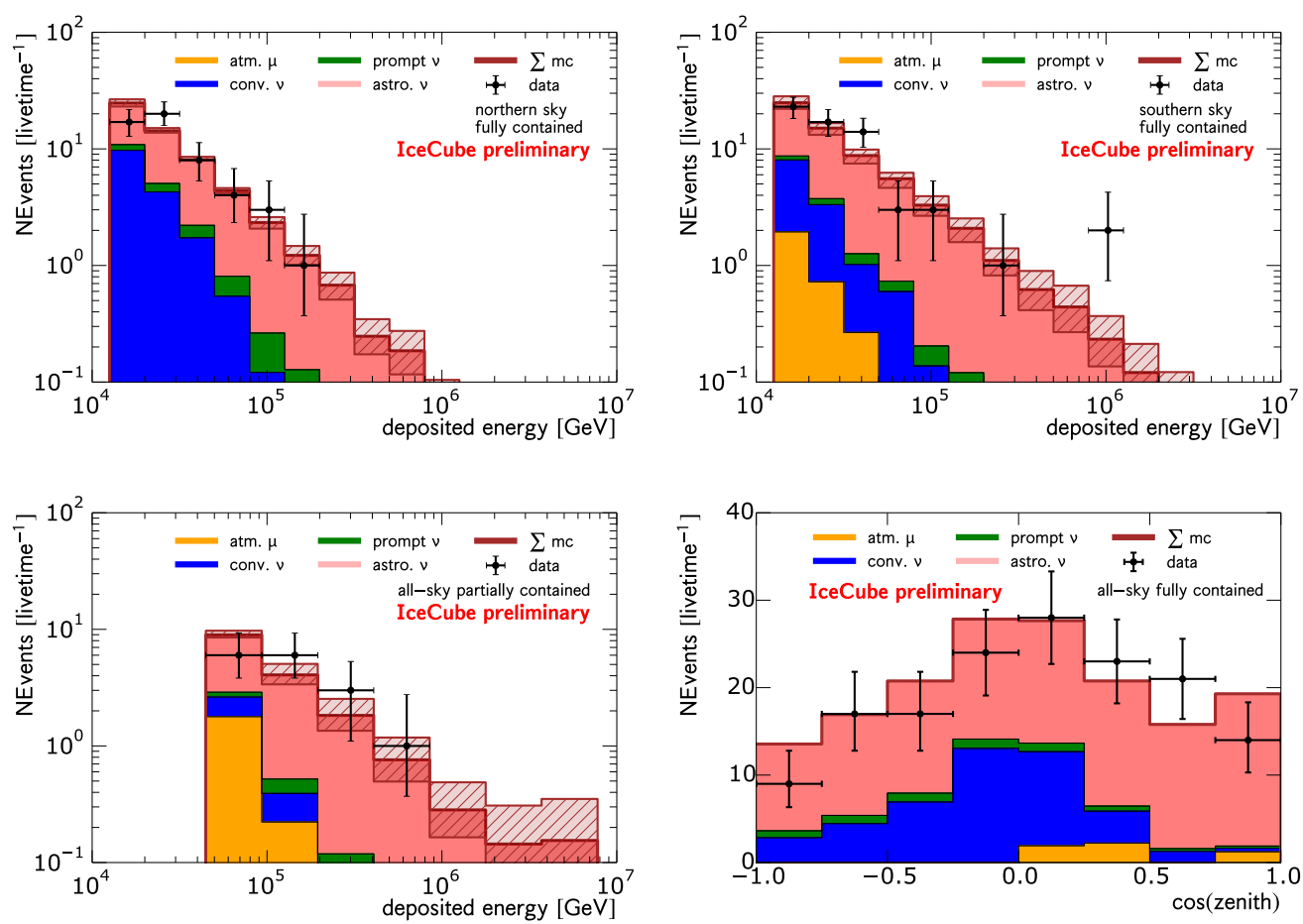

Figure 3: Energy distributions at final selection level: fully contained cascades, Northern sky (top left), Southern sky (top right), all-sky partially contained cascades (bottom left), hatched regions show $1 \sigma$ uncertainty on sum of m.c.; zenith distribution for fully contained cascades (bottom right)

from the North due to the atmospheric self-veto effect that breaks the spectral degeneracy between the prompt neutrino flux with the soft astrophysical flux we observed. This measurement also disfavors a hard spectral index of $\gamma=-2$. In this case we find a small astrophysical contribution $\phi=6 \cdot 10^{-19} \mathrm{GeV}^{-1} \mathrm{~s}^{-1} \mathrm{sr}^{-1} \mathrm{~cm}^{-2}$ that requires a rather large prompt component of $\phi_{\text {prompt }}=5 \cdot \Phi_{E R S}$. Such a combination is rejected at $3.5 \sigma$ based on the sampling distribution of the corresponding likelihood ratio test-statistic under this ' $E^{-2}$-hypothesis'. The isotropy assumption about the astrophysical flux could be challenged by looking for spectral differences between the fluxes measured from the Northern and Southern skies. Due to their better angular resolution compared to partially contained cascades, we constrain this investigation to contained cascades only. Here we perform the same maximum likelihood estimation for the samples from the Northern and Southern skies separately. For upward oriented showers we find the same parameters as for downward oriented showers $\gamma_{\text {north }}=2.69_{-0.34}^{+0.34}, \gamma_{\text {south }}=2.68_{-0.22}^{+0.20}$ and $\phi_{\text {north }}=1.7_{-1.2}^{+1.3} \mathrm{GeV}^{-1} \mathrm{~s}^{-1} \mathrm{sr}^{-1} \mathrm{~cm}^{-2}, \phi_{\text {south }}=1.9_{-0.6}^{+0.8} \mathrm{GeV}^{-1} \mathrm{~s}^{-1} \mathrm{sr}^{-1} \mathrm{~cm}^{-2}$ within large uncertainties due to the spectral degeneracy with the prompt normalization. This consistency remains when all nuisance parameters are kept fixed at the values obtained by the joint fit. For fixed nuisance parameters we find insignificantly higher astrophysical normalizations in very good agreement with the joint result. Any deviation of the data from the single power-law model may provide valuable insight into the nature of the observed astrophysical flux. Although we found no evidence for inconsistency between data and model, we did perform an alternative fit based on a 

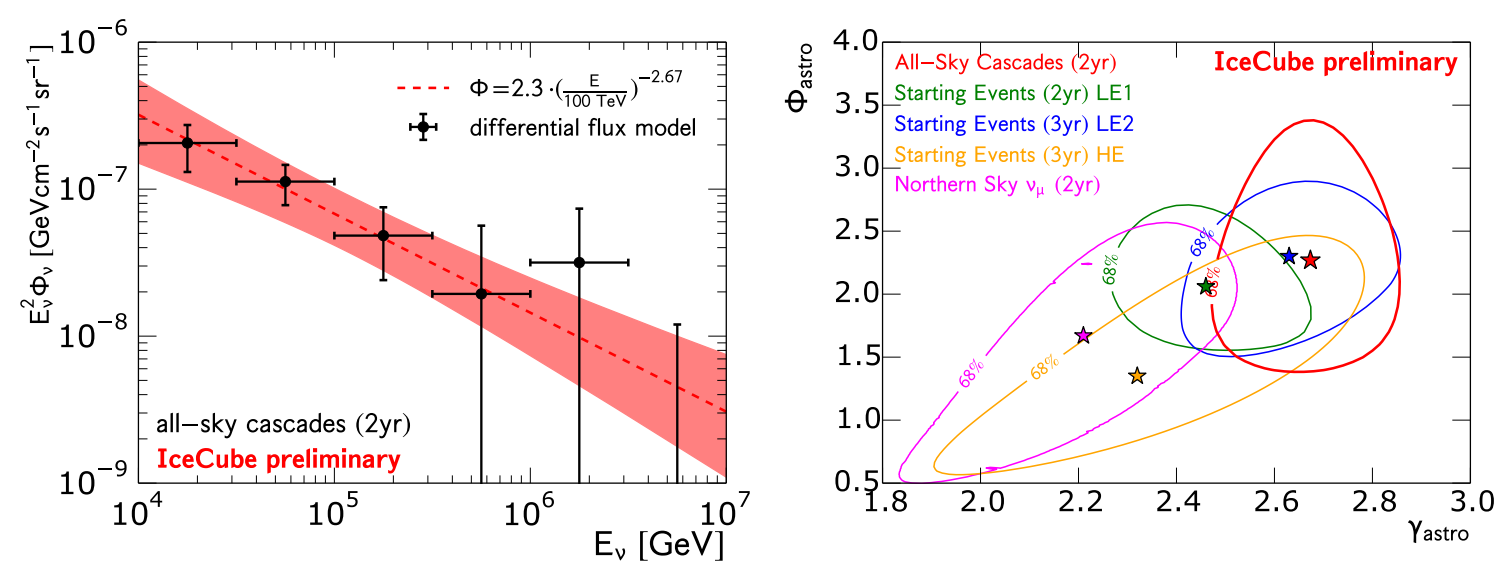

Figure 4: Left: best-fit astrophysical- $v$ flux (dashed line, red) and $1 \sigma$ band (red) compared to the "differential model" fit; Right: Comparison of recent $v$-flux measurements in IceCube: this analysis (red), ref. [5] (green), ref. [3] (purple), ref. [10] (blue) and ref. [11] (yellow).

"differential model" to measure the energy dependence of the observed flux. Similar to ref. [3] we extract the flux normalization in bins of neutrino energy $E_{v}$. Within each bin a $E^{-2}$-distribution is assumed. The corresponding expected distributions of the deposited energy serve as a basis to describe the data as linear combination on top of the expected background from atmospheric neutrinos and muons. The result (black) is shown in Fig. 4 (left) and compares very well to the power law model fit (red). The amount of overlap between this cascade-based event selection with the samples from the starting event analyses $[3,5,10]$ was found to be smaller than $\sim 25 \%$. There is no overlap with the Northern sky $v_{\mu}$-sample [11]. The measurement of the astrophysical flux parameters presented here can thus be regarded as approximately independent and is found to agree well with previous IceCube measurements (refs. [3, 5, 10]) as shown in Fig. 4. This was quantified to be better than $1 \sigma$ by applying Wald's method [24] in a bivariate normal approximation using the observed information matrices as estimates of the respective covariance matrices. This result seems to be in insignificant $(<2 \sigma)$ tension with the $v_{\mu}$ measurement [11] (Northern sky). Future data will help clarify the situation. We would like to point out that uni-variate comparisons of multi-variate measurements (e.g. "looking at $\gamma$ only") can lead to erroneous inference about consistency, as no statement about the other parameters is made.

Summary We have performed the first high energy $(E>10 \mathrm{TeV})$ cascade-only measurement of the diffuse astrophysical neutrino flux recently discovered by IceCube using 641 days of IceCube data (2010-2012). For the first time we extend topological cascade selection criteria to also include partially contained cascades with vertices in near proximity to the detector. This analysis found 172 cascade events of which approximately $60 \%$ ( $75 \%$ above $100 \mathrm{TeV})$ have not previously been reported by IceCube. The measured astrophysical component is well described by a smooth and featureless power-law. The preliminary fit result is a soft spectral index of $\gamma=2.67_{-0.13}^{+0.12}$ and a per-flavor normalization of $\phi=\left(2.3_{-0.6}^{+0.7}\right) \cdot 10^{-18} \mathrm{GeV}^{-1} \mathrm{~s}^{-1} \mathrm{sr}^{-1} \mathrm{~cm}^{-2}$ at $E=100 \mathrm{TeV}$ in agreement with the previous IceCube measurements. This sample can not be 
explained by atmospheric backgrounds, which would require a prompt neutrino contribution 7 times larger than expected and is rejected with a significance of $4.7 \sigma$. Similarly this sample disfavors a harder spectrum with $\gamma=-2$ at $3.5 \sigma$. We further measure the same astrophysical fluxes from the Northern $\left(\gamma_{\text {north }}=2.69_{-0.34}^{+0.34}\right)$ and Southern skies $\left(\gamma_{\text {south }}=2.68_{-0.22}^{+0.20}\right)$. This is consistent with the isotropic expectation for extra-galactic sources.

\section{References}

[1] A. Achterberg et al., Astropart. Phys. 26, 155 (2006).

[2] R. Abbasi et al., Phys. Rev. D 86, 022005 (2012).

[3] M. G. Aartsen et al., Science 342, 1242856 (2013);

M. G. Aartsen et al., Phys. Rev. Lett. 113, 101101 (2014).

[4] E. Waxman and J. Bahcall, Phys. Rev. D 59, 023002 (1998)

[5] M. G. Aartsen et al., Phys. Rev. D 91, 022001 (2015).

[6] E. Waxman and J. Bahcall, Phys. Rev. Lett. 78, 2292 (1997); K. Murase, S. Inoue, and S. Nagataki, Astrophys. J 689, L105 (2008); F. W. Stecker, Phys. Rev. D 88, 047301 (2013)

[7] T. K. Gaisser, F. Halzen, and T. Stanev, Physics Reports 258, 173 (1995)

[8] H. Athar, M. Jezabek and O. Yasuda, Phys. Rev. D 62, 103007 (2000)

[9] T. Kashti and E. Waxman, Phys. Rev. Lett. 95, 181101 (2005); P. Lipari, M. Lusignoli and D. Meloni, Phys. Rev. D 75, 123005 (2007); S. Choubey and W. Rodejohann, Phys. Rev. D 80, 113006 (2009)

[10] M. G. Aartsen et al., Phys. Rev. Lett. 114, 171102 (2015)

[11] M. G. Aartsen et al., Evidence for Astrophysical Muon Neutrinos from the Northern Sky with IceCube, submitted to Phys. Rev. Lett. (2015).

[12] M. Lesiak-Bzdak and A. Stoessl (for the IceCube Collab.), Proc. of the 33nd ICRC, paper 0370 (2013)

[13] D. Heck et al., CORSIKA, Tech. Rep. FZKA 6019, Forschungszentrum Karlsruhe (1998)

[14] T. Gaisser, Astroparticle Physics, Vol. 35, 12 (2012)

[15] M. Honda et al., Phys. Rev. D75, 043006 (2007)

[16] R. Enberg, M. H. Reno and I. Sarcevic, Phys. Rev. D78, 043005 (2008)

[17] M. G. Aartsen et al., Phys. Rev. D 89, 062007 (2014)

[18] T. Gaisser, K. Jero, A. Karle, J. van Santen, Phys. Rev. D 90, 023009 (2014)

[19] S. S. Wilks, Ann. Math. Statist. Vol. 9, 1 (1938)

[20] A. Fedynitch, J. Becker Tjus and P. Desiati, Phys. Rev. D 86, 114024 (2012)

[21] R. Abbasi et al., Nucl. Instr. Meth. Phys. Res. A, Vol. 618, 1-3 (2010)

[22] M. G. Aartsen et al., Nucl. Instr. Meth. Phys. Res. A, Vol. 711 (2013)

[23] S. Baker and R. D. Cousins, Nucl. Instr. Meth. Phys. Res., Vol. 221, 2 (1984)

[24] A. Wald, Trans. Amer. Math. Soc. 54 (1943); T. W. F. Stroud, Ann. Math. Stat., Vol. 42, No. 4 (1971) 